\title{
Intensity-resolved IR multiple photon ionization and fragmentation of $\mathrm{C}_{60}$
}

\author{
Joost M. Bakker, ${ }^{1}$ Vivike J.F. Lapoutre, ${ }^{1}$ Britta Redlich, ${ }^{1}$ Jos Oomens, ${ }^{1}$ Boris G. Sartakov, ${ }^{2}$ \\ André Fielicke, ${ }^{3}$ Gert von Helden, ${ }^{3}$ Gerard Meijer, ${ }^{3}$ and Alexander F. G. van der Meer ${ }^{1}$ \\ ${ }^{1}$ FOM Institute for Plasma Physics Rijnhuizen, \\ Edisonbaan 14, NL-3439 MN Nieuwegein, The Netherlands \\ ${ }^{2}$ A.M. Prokhorov General Physics Institute, \\ RAS, Vavilov Street 38, 119991 Moscow, Russia \\ ${ }^{3}$ Fritz-Haber-Institut der Max-Planck-Gesellschaft, \\ Faradayweg 4-6, D-14195 Berlin, Germany
}

\begin{abstract}
The sequential absorption of multiple infrared (IR) photons by isolated gas-phase species can lead to their dissociation and/or ionization. Using the newly constructed "Free Electron Laser for Intra-Cavity Experiments" (FELICE) beam line at the FELIX facility, neutral $\mathrm{C}_{60}$ molecules have been exposed to an extremely high number $\left(\approx 10^{23}\right)$ of photons $/ \mathrm{cm}^{2}$ for a total time duration of up to $5 \mu \mathrm{s}$. At wavelengths around $20 \mu \mathrm{m}$, resonant with allowed IR transitions of $\mathrm{C}_{60}$, ionization and extensive fragmentation of the fullerenes is observed. The resulting photofragment distributions are attributed to absorption in fragmentation products formed once $\mathrm{C}_{60}$ is excited to internal energies at which fragmentation or ionization takes place within the duration of the laser pulse. The high IR intensities available combined with the large interaction volume permit spatially resolved detection of the ions inside the laser beam, thereby disentangling the contributions from different IR intensities. The use of spatial imaging reveals intensity dependent mass distributions that are substantially narrower than what has been observed previously, indicating rather narrow energy distributions. A simple rate-equation modeling of the excitation process supports the experimental observations.
\end{abstract}




\section{INTRODUCTION}

$\mathrm{C}_{60}$ is a well-studied model system for competing statistical energy release mechanisms in finite systems ${ }^{1,2}$. In highly excited $\mathrm{C}_{60}$ energy release in the form of ionization (thermionic emission), fragmentation and radiative emission have been observed ${ }^{3-8} \cdot \mathrm{C}_{60}$ has also played an important role in the understanding of infrared (IR) multiple-photon excitation (IR-MPE) spectroscopy. Using tightly focused tunable IR radiation from the free-electron laser FELIX, it was demonstrated in 1997 that neutral $\mathrm{C}_{60}$ can be resonantly heated by the absorption of multiple IR photons to an extent that it undergoes thermionic electron emission ${ }^{9}$. For this to be observed on the time-scale of that particular experiment, internal energies of around 50 $\mathrm{eV}$ are needed ${ }^{10}$, implying absorption of several hundreds of IR photons. In between absorptions the energy is re-distributed over all internal degrees of freedom through intramolecular vibrational energy redistribution $(\mathrm{IVR})^{11}$. The resulting so-called IR-Resonance Enhanced Multiple Photon Ionization (IR-REMPI) spectrum showed the four electric dipole allowed IR transitions as well as a higher-frequency IR-active combination band of $\mathrm{C}_{60}$. By modeling the excitation mechanism with a set of coupled rate equations a quantitative understanding of the observed spectral lineshapes and their relative intensities has been obtained ${ }^{12,13}$.

A remarkable observation in these experiments was that the resulting mass spectrum is very clean, i.e. virtually no fragmentation is observed. Upon excitation around the lowest frequency $F_{1 u}$ mode at $515 \mathrm{~cm}^{-1}$ in particular, almost exclusively $\mathrm{C}_{60}^{+}$parent ions are observed. This was taken as evidence that the number of photons absorbed approximately follows a Poisson distribution, with a relative width given by $1 / \sqrt{N_{\text {avg }}}$, where $N_{\text {avg }}$ is the average number of photons absorbed by the molecules ${ }^{14}$. As the number of photons needed scales inversely with the photon energy, it follows that the distribution becomes very narrow at low photon energies. In practice, in an IR-MPE experiment, the effect of the narrow internal energy distribution at a well-defined fluence is often masked by the rather broad distribution of fluences that the molecules experience when they pass through the Gaussian profile of the laser beam ${ }^{15}$. Nevertheless, it was anticipated that rather peculiar fragment-ion distributions could be produced at yet higher IR laser fluences, but these were not available at the time. In the present study we demonstrate IR multiple photon excitation of neutral $\mathrm{C}_{60}$ under conditions that combine higher fluences of IR radiation with a substantially larger interaction region. This is achieved by employing the newly constructed "Free Electron Laser 
for Intra-Cavity Experiments" (FELICE) beam line at the FELIX facility ${ }^{16}$. Both volumeintegrated and spatially resolved fragment-ion distributions have been measured from the interaction region with the IR-FEL beam.

\section{EXPERIMENT}

\section{A. Experimental apparatus}

The IR user facility FELIX has been extended with a new beam line "FELICE" (Free Electron Laser for Intra-Cavity Experiments). This beam line is devoted to gas-phase experiments requiring very high IR intensities. In order to achieve maximal intensity the experiments are integrated in the FELICE resonator which consists of a four mirror cavity. This configuration provides up to a factor of 100 higher IR pulse energy at the experiment than the conventional FELIX beam lines. The versatile molecular beam and cluster instrument, installed in the 2-m section between the last two mirrors of the cavity, is shown in an artist's impression in Figure 1a, with a schematic overview of the various source and interaction chambers in Figure 1b. The instrument is designed to enable an effective use of the FELICE beam time. It consists of a vacuum chamber with two identical interaction regions spaced $300 \mathrm{~mm}$ apart, each fitted with several vacuum flanges.

Interaction chamber 1 is equipped with a reflectron time-of-flight (RETOF, Jordan TOF Products, Inc.) mass spectrometer (vertical extraction), using a MultiChannel Plate (MCP) detector. The typical mass resolution is $M / \Delta M \approx 1700$. Two source chambers are attached to this interaction chamber, each of which has two differentially pumped chambers. Molecular beams produced in these source chambers enter the interaction chamber through a gate valve allowing for sample changing while using the other source. The molecular beams run in the horizontal plane, are mutually perpendicular and cross the FELICE beam at angles of $35^{\circ}$ and $55^{\circ}$, respectively. Both source chambers are designed to accommodate different molecular beam sources mounted on standard vacuum flanges.

Interaction chamber 2 is currently fitted with an imaging spectrometer consisting of an open-electrode assembly (vertical extraction) and a MCP detector with a fast phosphor screen ${ }^{17}$. For the present experiments, a small chamber with an effusive beam source is directly attached to this interaction chamber. 
To minimize the length of the FELICE cavity, which extends below the experimental hall, the laser beam height with respect to the floor is only $400 \mathrm{~mm}$. Spatial overlap between molecular beam and laser beam is obtained by translating the molecular beam setup along the vertical axis under computer control. Furthermore, the experiment can be translated along the laser axis up to $300 \mathrm{~mm}$ from the focus resulting in a power density variation of a factor of 30. This movement also allows switching between the interaction chambers.

At the experiment, the optical beam is near-Gaussian and characterized by a Rayleigh range of $55 \mathrm{~mm}$ and a focus position at the center of the $2-\mathrm{m}$ section. With a typical micropulse energy of $0.5 \mathrm{~mJ}$, the intensity is on the order of $8 \cdot 10^{11} / \lambda \tau \mathrm{Wcm}^{-2}$ where $\lambda$ is the wavelength in $\mu \mathrm{m}$ and $\tau$ the FWHM pulse length in ps, which for the current experiment is approximately $0.1 \lambda$. The maximum achievable power density is $3 \cdot 10^{13} / \lambda^{2} \mathrm{~W} / \mathrm{cm}^{2}$

FEL laser pulses are produced in a pulse train, the so-called macropulse, with a typical duration of $\approx 5 \mu \mathrm{s}$, consisting of ps-long micropulses at a $1 \mathrm{~ns}$ separation. The optical pulse at the interaction point consists of two pulse trains that are shifted from 0 to 0.5 ns with respect to each other depending on the position along the laser axis.

The radiation is near transform-limited and the spectral width can be adjusted between typically $0.4 \%$ and $2 \%$ FWHM of the central frequency. The total energy of the macropulse is monitored from a small fraction of the light that is coupled out via a central hole in one of the end mirrors; macropulse energies up to $5 \mathrm{~J}$ have been deduced. With a macropulse duration of $5 \mu \mathrm{s}$, the micropulse energy is $1 \mathrm{~mJ}$ in that case. For the experiments described here typical macropulse energies used are about $2 \mathrm{~J}$. Molecular samples inside the cavity that interact with the full macropulse observe each micropulse twice, namely traveling to and from the end mirror.

The laser is linearly polarized in the vertical plane. The wavenumber range explored in our experiments is $250-2000 \mathrm{~cm}^{-1}$. An overview of the FELICE specifications relevant to these experiments is given in Table I. Further details of the FELICE beam line will be communicated in a forthcoming publication.

\section{B. $\mathbf{C}_{60}$ experiments}

Experiments on the IR excitation of $\mathrm{C}_{60}$ are performed using two detection schemes: the RETOF mass spectrometer mounted in interaction chamber 1 and the imaging spectrom- 
eter in interaction chamber 2. For the experiments with the RETOF, the source chamber is equipped with an effusive beam source. $\mathrm{C}_{60}$ powder (99\% pure, MER corporation) is sublimed at a temperature of $\approx 500{ }^{\circ} \mathrm{C}$ in a graphite oven with a $1 \mathrm{~mm}$ diameter aperture to form an effusive beam. The beam is collimated by a $1 \mathrm{~mm}$ diameter aperture $50 \mathrm{~mm}$ downstream of the oven.

Approximately $10 \mu$ s after interaction with the FELICE macropulse the ions formed are pulse extracted and mass-analyzed in the RETOF mass spectrometer. The ion current produced by the MCP is integrated and recorded with a digitizer (Acqiris DP310).

In the imaging experiments a similar effusive beam source is directly attached to interaction chamber 2 . The beam is shaped by a $0.3 \mathrm{~mm}$ high horizontal slit located $75 \mathrm{~mm}$ upstream from the point of interaction with FELICE and runs perpendicular to the FELICE beam. All ions are pulse-extracted within $1 \mu$ s after the FELICE macropulse towards an MCP detector using three circular electrodes of which the upper two have circular holes (no meshes) for ion transmission, as is commonly used for velocity map imaging (VMI $)^{18}$. Unlike in VMI the voltages are chosen such that the ion spatial distribution is directly projected onto the MCP. The used acceleration energy is $\approx 4 \mathrm{keV}$. The electrons produced in the MCP are accelerated towards a fast phosphor screen (P46). The phosphor screen is then recorded using an intensified CCD camera (Andor iStar) with the intensifier gated on over periods of $50 \mathrm{~ns}$ to allow for mass resolved detection of the ions. The mass resolution of the imaging detector is $M / \Delta M \approx 150$, allowing full temporal separation of ion fragments that typically are separated by a $\mathrm{C}_{2}(\mathrm{~m}=24 \mathrm{amu})$ unit.

\section{RESULTS}

\section{A. Spectral information}

The IR-REMPI spectrum of $\mathrm{C}_{60}$ measured with the intracavity setup (Figure $2 \mathrm{~b}$ ), is obtained by summing the volume-integrated ion distribution (all masses) that results from irradiation of neutral $\mathrm{C}_{60}$ molecules as a function of wavelength. The FELICE spectrum (panel b) is compared to the spectrum recorded using FELIX (from Ref. 9, panel a) . In the latter spectrum the four electric dipole allowed IR fundamental transitions of the $\mathrm{C}_{60}$ molecule and one combination mode are observed. These transitions are also observed in the 
FELICE spectrum at identical frequencies but with substantial broadening. Interestingly, the higher IR fluences available in FELICE allow for an efficient excitation of at least six additional resonances. Especially the observation of the spectrally narrow resonance just above $900 \mathrm{~cm}^{-1}$ demonstrates that the increased IR intensities and prolonged interaction with FELICE permit detection of modes with substantially lower IR transition strength. It is of interest to note that in this spectral range, IR-REMPI has been observed previously with a line-tuneable $\mathrm{CO}_{2}$ laser (at 941, 981, 1037 and $1076 \mathrm{~cm}^{-1}$, respectively) albeit at substantially higher fluences ${ }^{5}$. All newly found resonances can readily be assigned to combination modes that have been observed in thick-film IR absorption measurements ${ }^{19}$. By reducing the power density in the current experiment by a factor of about 20 the upper spectrum, as recorded with FELIX, is reproduced.

\section{B. Volume integrated mass spectra}

Figure 3 shows typical mass spectra of the ion distribution resulting from irradiation of $\mathrm{C}_{60}$ at 515,559 and $1385 \mathrm{~cm}^{-1}$, respectively, with macropulse energies of $\approx 2 \mathrm{~J}$. These wavenumbers correspond to IR allowed fundamental vibrational transitions previously observed with IR-REMPI ${ }^{9}$ and indicated with an asterisk in Figure 2. Ions of all masses associated with $\mathrm{C}_{60-2 n}(\mathrm{n}=0,1,2, . ., 15)$ are observed, in accordance with the generally accepted process of sequential $\mathrm{C}_{2}$ loss all the way down to $\mathrm{C}_{30}$, the smallest observed fullerene ion $^{20,21}$. The small signal peaks in between the strong $\mathrm{C}_{60-2 n}$ peaks (marked with an asterisk in Figure 3) result from delayed fragmentation in the flight tube. In the mass spectra recorded at 559 and $1385 \mathrm{~cm}^{-1}$, respectively, small linear and cyclic fragments appear as well. These bimodal mass spectra are consistent with previous observations after laser excitation of $\mathrm{C}_{60}{ }^{4,5,22}$. In contrast to short-pulse laser excitation experiments, where a phase transition-like behavior is expected to occur at internal temperatures of 4000-5000 K, ${ }^{23}$ it appears unlikely that such high internal temperature are reached here (see the Discussion section below). The smaller fragments observed here likely result from further fragmentation of smaller fullerenic fragment ions after re-absorption of IR light.

The distribution does not have a continuous functional shape, but certain stoichiometries, such as $\mathrm{C}_{56}^{+}, \mathrm{C}_{50}^{+}, \mathrm{C}_{44}^{+}$and $\mathrm{C}_{36}^{+}$, are favored. These ions are known from mass-spectrometry experiments to be energetically more stable than the neighboring ions ${ }^{24}$. 
The smallest fullerene fragment ion observed, $\mathrm{C}_{30}^{+}$, allows a rough estimate of the internal energy reached and the corresponding number of photons absorbed. For thermionic emission in $\mathrm{C}_{60}$ to take place within the experimental time window, statistical models predict a required internal energy on the order of $50 \mathrm{eV}^{10}$. The appearance of $\mathrm{C}_{58}^{+}$requires an additional internal energy of $\approx 10.8 \mathrm{eV}$, the fragmentation energy for $\mathrm{C}_{2}$ evaporation from $\mathrm{C}_{60}^{+10}$, leading to an estimated internal energy of $\approx 60 \mathrm{eV}$. By extrapolating the dissociation sequence progression to the fragmentation of $\mathrm{C}_{32}^{+}$into $\mathrm{C}_{30}^{+}$the energy absorbed is estimated to be in excess of $150 \mathrm{eV}$, equivalent to more than 2000 photons at $20 \mu \mathrm{m}$.

Interestingly, the mass distribution after irradiation at $559 \mathrm{~cm}^{-1}$, is shifted towards lower mass fragments than after excitation at $515 \mathrm{~cm}^{-1}$, coinciding with the strongest IR active mode in the linear spectrum of $\mathrm{C}_{60}$. Therefore, the evolution of the mass spectra as a function of the IR intensity was recorded at various distances from the laser focus. In Figure 4, the first column shows the IR-REMPI spectrum for $\mathrm{C}_{60}$ obtained by summing the total ion yield. The second and third columns show a comparison of the mass spectra resulting from irradiation at 515 and $559 \mathrm{~cm}^{-1}$, respectively. In the action spectra the $515 \mathrm{~cm}^{-1}$ mode appears at substantially lower fluences than the $559 \mathrm{~cm}^{-1}$ mode. Saturation of the signal for the first mode occurs at a few percent of the maximum available intensity, when the 559 $\mathrm{cm}^{-1}$ mode is still hardly visible. However, with increasing laser intensity the fragmentation for the $559 \mathrm{~cm}^{-1}$ mode rapidly increases and is even more extensive in the focus than is the case for the $515 \mathrm{~cm}^{-1}$ mode. It should be noted that the total ion yield reaches a maximum at intermediate intensities. The fact that the total yield starts to decrease again when the experiment approaches the focus position is explained by the reduction of the number of molecules within the interaction volume in combination with a near $100 \%$ fragmentation and/or ionization efficiency.

\section{Spatially resolved mass spectra}

All data presented in Figures 3 and 4 are recorded using the RETOF mass spectrometer. This instrument integrates the signal of all ions that are formed in the interaction volume defined by the overlap between molecular beam and laser beam. The recorded mass spectra result from a convolution of the molecular beam density and velocity profile and the Gaussian laser profile, complicating their straightforward interpretation. This difficulty can partly be 
overcome by using the technique of spatial map imaging (SMI) which allows to measure the distribution of the ions as projected onto the plane defined by the laser beam and molecular beam ${ }^{17}$ and thus the ion distribution as a function of distance from the laser beam axis.

To reduce the contribution from molecules above and below the plane of intersection, a slit aperture is placed in the path of the molecular beam, confining the height to roughly $0.5 \mathrm{~mm}$ at the interaction point with FELICE, whereas the FWHM of the intensity profile of the optical beam at the focus is $\approx 1 \mathrm{~mm}$ at $500 \mathrm{~cm}^{-1}$. Along the axis of the laser beam the width of the molecular beam is roughly $10 \mathrm{~mm}$. Calibration of the spatial coordinates is done by recording images of $\mathrm{C}_{60}^{+}$ions produced by a tightly focused $\mathrm{ArF}$ excimer laser (193 nm) at several positions.

In Figure 5a, a spatially resolved measurement at $528 \mathrm{~cm}^{-1}$, where the fragmentation is somewhat stronger than at $515 \mathrm{~cm}^{-1}$, is presented. This image is recorded $55 \mathrm{~mm}$ from the FELICE focus where the laser beam area is twice the value at the focus. Panel (a) shows a raw image of the detected $\mathrm{C}_{60}^{+}$ion distribution. An elongated distribution along the laser propagation axis is observed. At the left and right ends the distribution is cut off by the detector dimensions. Aberrations in the electrode lens system are responsible for the slight curvature along the laser axis. The main feature is the splitting of the distribution into two distinct regions where $\mathrm{C}_{60}^{+}$ions are detected. The two regions are not centered around the laser axis, due to the extraction after the $5 \mu$ s duration macropulse combined with the molecular beam speed ( $c f$. Figure 8, discussed below). The regions where $\mathrm{C}_{60}^{+}$ions are detected correspond to regions of low intensity at the edges of the laser beam where $\mathrm{C}_{60}^{+}$ions are formed. This is not unreasonable given that $\mathrm{C}_{60}^{+}$ions form at relatively low intensities, where ionization but no further fragmentation can be induced, as shown in Figure 4.

To extract intensity dependent ion fragment distributions, the images recorded are integrated along the laser propagation axis. An example of an integrated spatial distribution is shown in Figure 5b. To reduce the effect of aberrations only the central part of the images is taken into account, as indicated by the square in Figure 5. Applying the same integration procedure, position-resolved time-of-flight (TOF) mass spectra can be obtained by gating the detector on at different delay times after extraction. Mass spectra for the two closely spaced resonances at $528 \mathrm{~cm}^{-1}$ and $559 \mathrm{~cm}^{-1}$ were recorded at a $55 \mathrm{~mm}$ distance from the focus (Figure 6) and at the focus itself (Figure 7). The strong effect that a doubling of the power density has on the shape of the mass spectra is apparent, especially at $559 \mathrm{~cm}^{-1}$. As 
expected, the smaller fragments are measured near the laser axis and the larger ones at larger radial distances. In the measurements recorded at $55 \mathrm{~mm}$ from the focus, rather narrow ion distributions, peaking at $\mathrm{C}_{50}^{+}$, can be observed, whereas in the focus the distributions are much broader. The fact that the $\mathrm{C}_{60}^{+}$signal on axis is stronger at the focus than at $55 \mathrm{~mm}$ is likely explained by the fact that the height of the molecular beam $(\approx 0.5 \mathrm{~mm})$ is no longer substantially smaller than the beam waist $(0.6 \mathrm{~mm})$ near the focus.

\section{Simulation model}

To obtain a better understanding of the effect of the various experimental parameters, the absorption in $\mathrm{C}_{60}$ was modeled at an excitation frequency of $528 \mathrm{~cm}^{-1}$. The computational algorithm has been described in detail earlier ${ }^{12-14}$ and only some key aspects are given here. In the statistical model the internal energy of the $\mathrm{C}_{60}$ molecules after interacting with a FELICE macropulse is calculated by solving the rate equations

$$
\begin{aligned}
d n_{i} / d t= & k_{i, i+1} n_{i+1}+k_{i, i-1} n_{i-1} \\
& -k_{i+1, i} n_{i}-k_{i-1, i} n_{i} \\
& -k_{d i s s}\left(T_{e f f, i}\right) n_{i}-k_{i o n}\left(T_{e f f, i}\right) n_{i}
\end{aligned}
$$

with $n_{i}$ the number of molecules having absorbed $i$ photons, $k_{i, j}$ the transition rate from state $j$ to state $i$ and $k_{d i s s}\left(T_{e f f, i}\right)$ and $k_{i o n}\left(T_{e f f, i}\right)$ the dissociation and ionization rates at an effective temperature $T_{e f f, i}$, corresponding to the energy of the molecules in state $i$. For the evaluation of the effective temperature, the anharmonicity is assumed to be a small perturbation, so that it can be evaluated using the equation

$$
E_{i}=\sum_{m=1}^{3 N-6} \frac{\hbar \omega_{m}}{\exp \left(\hbar \omega_{m} / k T_{e f f, i}\right)-1}
$$

with $\omega_{m}$ the frequency of the $m$-th vibrational mode.

The rate equations are solved for a $5 \mu$ s duration macropulse with a square wave intensity distribution in time (thereby neglecting the micropulse structure) with the same average power as used in the experiment. The dissociation and ionization rates $k_{d i s s}\left(T_{e f f, i}\right)$ and $k_{i o n}\left(T_{e f f, i}\right)$ are calculated using the parameters given by Tomita et al. ${ }^{10}$. It must be stressed that such simulations can only be done when the absorption properties of the molecules, including IR absorption cross-sections and anharmonicity parameters, are well 
known. Therefore, realistic simulations can only be carried out for the excitation process in neutral $\mathrm{C}_{60}$; insufficient knowledge on the absorption properties of the fragments prohibits a quantitative analysis of their spatial distributions.

The simulated profile of the $\mathrm{C}_{60}^{+}$ion distribution along the laser profile corresponding to the measurements of Figure 5 is shown in Figure 8b. The laser geometry is assumed to be Gaussian with a $55 \mathrm{~mm}$ Rayleigh range and the velocity distribution of the molecular beam to be Maxwellian corresponding to the oven temperature of $500^{\circ} \mathrm{C}$. The distribution of $\mathrm{C}_{60}^{+}$ions was obtained by summing over all molecules having an energy in the range $\left[E_{a p p, C_{60}^{+}}, E_{a p p, C_{60}^{+}}+E_{\text {diss }, C_{60}^{+}}\right]$, where $E_{\text {diss }, C_{60}^{+}}$is the dissociation energy of $C_{60}^{+}$, taken from

Ref 10 while $E_{a p p, C_{60}^{+}}$, the appearance energy for $C_{60}^{+}$, is used as fitting parameter. The value giving the best fit for the appearance energy, $53 \mathrm{eV}$, compares favorably with the value at which the ionization rate exceeds $10^{6}$ per sec. ${ }^{10}$, i.e. the timescale of the experiment. The broadening and asymmetry resulting from the thermal velocity can be inferred from (c) and (d), where the computed distributions are shown for molecules with constant velocities of 135 and $0 \mathrm{~m} / \mathrm{s}$, respectively.

It should be noted that there is a small difference in the shift of each peak in (c) relative to (d) due to the (sign of the) curvature in the intensity profile of the laser. It is this difference in shifts for the falling and rising edges of the laser beam profile, when integrated over all velocities present in the molecular beam, that gives rise to the asymmetry in the peaks in (a) and (b).

\section{DISCUSSION}

The ionization and dissociation rates of $\mathrm{C}_{60}$ grow exponentially with internal energy and above $60 \mathrm{eV}$ the dissociation rate (greatly) exceeds the micropulse repetition rate ${ }^{10}$. This means that under our experimental conditions it is impossible to pump substantially more than $50 \mathrm{eV}$ in $\mathrm{C}_{60}$ before it fragments. This is illustrated in Figure 9, where the internal energy of $\mathrm{C}_{60}$ is calculated as a function of laser intensity for a $5 \mu$ s duration macropulse for the case where ionization and fragmentation rates are included (triangles), and for the case where these are neglected (squares and circles). The fragmentation and ionization rates are calculated following Ref. 10. One sees that the internal energy never exceeds $53 \mathrm{eV}$ when ionization and fragmentation are taken into account. Since similar arguments are 
expected to hold for each of the fragments, it follows that they all must have an appreciable IR absorption cross-section in this spectral range, contrary to what we suggested previously based on the relative spectral isolation of this mode ${ }^{9,14}$. Partly this can be explained by the fact that the fragments are 'born' with high internal energy and will therefore be in a vibrational quasi-continuum, giving rise to non-resonant absorption. On the other hand, the markedly different intensity dependence of the fragment production at $559 \mathrm{~cm}^{-1}$ compared to $515 \mathrm{~cm}^{-1}$, shows that the fragments themselves also possess wavelength dependent IR cross sections in this spectral range. The fact that the spectrum at high internal energies is still markedly structured and alike to that at low internal energies is illustrated for $\mathrm{C}_{60}$ in the inset of Figure 9.

As expected, some of the observed fragment distributions are rather narrow but not quite as narrow as the width corresponding to the $\sqrt{N_{\text {avg }}}$ width of a Poisson distribution. For example, the top spectrum of Figure 6 a corresponds to a width $\sigma \approx 9 \mathrm{eV}$, whereas $\hbar \omega \sqrt{N_{\text {avg }}}$ equals $2.5 \mathrm{eV}$.

Part of the discrepancy observed is due to the omission of stimulated emission in the formula for the width of the Poisson distribution: the width should be equal to the square root of the average number of transitions, both up and down, made in reaching energy state i, i.e. not just the minimal number of steps needed. As an illustration, the computed energy distribution at an average energy of $86 \mathrm{eV}$ exhibits a width $\sigma=4.6 \mathrm{eV}$, i.e. twice the value one obtains when the number of photons involved is set to $86 \mathrm{eV}$ divided by the photon energy.

As Figure 8 suggests, an additional large contribution to the further broadening of the mass distribution is probably caused by blurring due to the thermal velocity distribution of the molecules, which leads to position scrambling: with a most probable velocity of 135 $\mathrm{m} / \mathrm{s}$, corresponding to an oven temperature of $500{ }^{\circ} \mathrm{C}$, the distance traveled in $5 \mu \mathrm{s}$ is $0.675 \mathrm{~mm}$, whereas the FWHM of the laser beam intensity distribution is $\approx 1 \mathrm{~mm}$ at the focus. Qualitatively, this blurring effect on the mass distribution can be simulated by setting $k_{\text {diss }}\left(T_{e f f, i}\right)$ and $k_{i o n}\left(T_{e f f, i}\right)$ to zero and compute the internal energy distribution of $\mathrm{C}_{60}$ as if there would be no losses due to fragmentation or ionization. This is done for the actual velocity distribution of the molecules and for the case where the molecules are stationary. The resulting energy distribution is then converted to a fragment distribution for $\mathrm{C}_{60-2 n}$ by summing over all molecules in the energy range $E_{a p p, C_{60-2 n}^{+}}$to $E_{a p p, C_{60-2 n-2}^{+}}$. The appearance 
energy for fragment $\mathrm{C}_{60-2 n}^{+}$is set equal to the appearance energy for $\mathrm{C}_{60}^{+}$plus the sum of the fragmentation energies of the fragments $\mathrm{C}_{60-2 m}^{+}$with $m<n$, taken from Ref. 24. The results for conditions similar to Figure 7a and Figure 6a are shown in Figures 10a and 10b respectively.

The cut through the distribution for the upper mass spectrum for each simulation is taken at the position where the smallest fragment has a maximum abundance; the other mass spectra are determined at positions relative to this cut that are equivalent to those indicated in Figures 7a and 6a, respectively. The effect of the thermal velocity distribution is clearly quite substantial, even at $55 \mathrm{~mm}$ from the focus. In view of the fact that the fragmentation must proceed via absorption in intermediate fragments, it is somewhat surprising that these simulations actually fit the measured fragment distributions fairly well.

\section{CONCLUSIONS}

In conclusion, we have observed massive fragmentation and ionization upon resonant IR excitation of neutral $\mathrm{C}_{60}$ molecules at the IR laser pulse energy densities that can be obtained with the newly constructed FELICE beam line at the FELIX facility. As the exponentially growing dissociation rate sets a hard limit in the range of $50-60 \mathrm{eV}$ (53 eV according to the simulations) for the internal energy of neutral $\mathrm{C}_{60}$ under our experimental conditions, it is concluded that the extensive fragmentation must be due to further excitation of decay products. Even though the fragments are very hot with typical internal energies of $40 \mathrm{eV}$, a significant change in fragmentation yield is observed when changing the excitation wavelength from $528 \mathrm{~cm}^{-1}$ to $559 \mathrm{~cm}^{-1}$. Owing to the relatively large size of the interaction volume, it is possible to use spatial map imaging to obtain intensity dependent mass-spectra. Observed fragment distributions, especially those obtained after excitation around $515 \mathrm{~cm}^{-1}$, where a vibrational mode reasonably unique to $\mathrm{C}_{60}$ is located, are consistent with the narrow internal energy distributions expected for IR-MPE at low photon energy and the position scrambling due to the thermal velocity (spread) of the molecules.

Acknowledgment This work is part of the research programme of the 'Stichting voor Fundamenteel Onderzoek der Materie (FOM)', and the construction of the FELICE beam line was funded by the 'Nederlandse Organisatie voor Wetenschappelijk Onderzoek' (NWO) 
through the NWO-Groot scheme.

1 E. E. B. Campbell and R. D. Levine, Annu. Rev. Phys. Chem. 51, 65 (2000).

2 J. U. Andersen, E. Bonderup, and K. Hansen, J. Phys. B- At. Mol. Opt. Phys. 35, R1 (2002).

3 E. E. B. Campbell, G. Ulmer, and I. V. Hertel, Phys. Rev. Lett. 67, 1986 (1991).

4 K. R. Lykke and P. Wurz, J. Phys. Chem. 96, 3191 (1992).

5 M. Hippler, M. Quack, R. Schwarz, G. Seyfang, S. Matt, and T. Mark, Chem. Phys. Lett. 278, 111 (1997).

6 E. E. B. Campbell, K. Hansen, K. Hoffmann, G. Korn, M. Tchaplyguine, M. Wittmann, and I. V. Hertel, Phys. Rev. Lett. 84, 2128 (2000).

7 F. Lepine and C. Bordas, Phys. Rev. A 69, 053201 (2004).

8 E. Kolodney, A. Budrevich, and B. Tsipinyuk, Phys. Rev. Lett. 74, 510 (1995).

9 G. von Helden, I. Holleman, G. M. H. Knippels, A. F. G. van der Meer, and G. Meijer, Phys. Rev. Lett. 79, 5234 (1997).

10 S. Tomita, J. U. Andersen, K. Hansen, and P. Hvelplund, Chem. Phys. Lett. 382, 120 (2003).

11 K. K. Lehmann, G. Scoles, and B. H. Pate, Annu. Rev. Phys. Chem. 45, 241 (1994).

12 G. von Helden, I. Holleman, G. Meijer, and B. Sartakov, Optics Express 4, 46 (1999).

13 G. von Helden, D. van Heijnsbergen, and G. Meijer, J. Phys. Chem. A 107, 1671 (2003).

14 A. Bekkerman, E. Kolodney, G. von Helden, B. Sartakov, D. van Heijnsbergen, and G. Meijer, J. Chem. Phys. 124, 184312 (2006).

15 K. Mehlig, K. Hansen, M. Heden, A. Lassesson, A. V. Bulgakov, and E. E. B. Campbell, J. Chem. Phys. 120, 4281 (2004).

16 http://www.rijnhuizen.nl/felix.

17 D. W. Chandler and P. L. Houston, J. Chem. Phys. 87, 1445 (1987).

18 A. Eppink and D. H. Parker, Rev. Sci. Instrum. 68, 3477 (1997).

19 K. A. Wang, A. M. Rao, P. C. Eklund, M. S. Dresselhaus, and G. Dresselhaus, Phys. Rev. B 48, 11375 (1993).

20 G. von Helden, M. T. Hsu, P. R. Kemper, and M. T. Bowers, J. Chem. Phys. 95, 3835 (1991).

21 G. von Helden, M. T. Hsu, N. Gotts, and M. T. Bowers, J. Phys. Chem. 97, 8182 (1993).

22 H. Hohmann, C. Callegari, S. Furrer, D. Grosenick, E. E. B. Campbell, and I. V. Hertel, Phys. 
Rev. Lett. 73, 1919 (1994).

23 E. E. B. Campbell, T. Raz, and R. D. Levine, Chem. Phys. Lett. 253, 261 (1996).

24 S. Tomita, J. U. Andersen, C. Gottrup, P. Hvelplund, and U. V. Pedersen, Phys. Rev. Lett. 87, 073401 (2001). 
Figures 

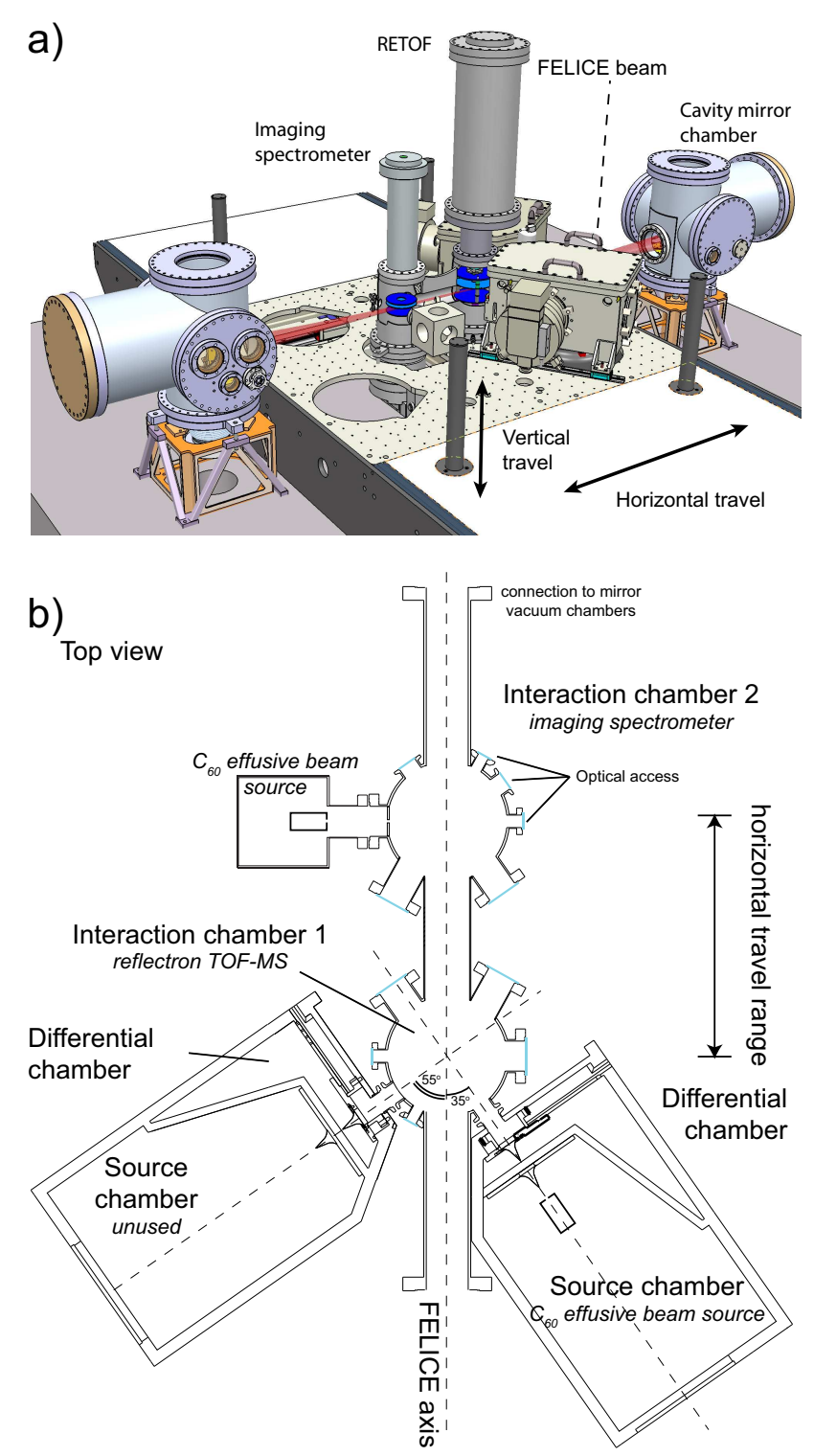

FIG. 1: An artist's impression of one of the experiments of the FELICE beam line, a molecular beam and cluster setup, is shown in panel a. The setup combines two FELICE interaction and detection chambers with up to four source chambers, two for each interaction chamber (see panel b). The experiments described are performed using (i) the effusive beam source and imaging detection in interaction chamber 2 or (ii) an effusive beam source in the source chamber connected to interaction chamber 1 and employing RETOF detection. 


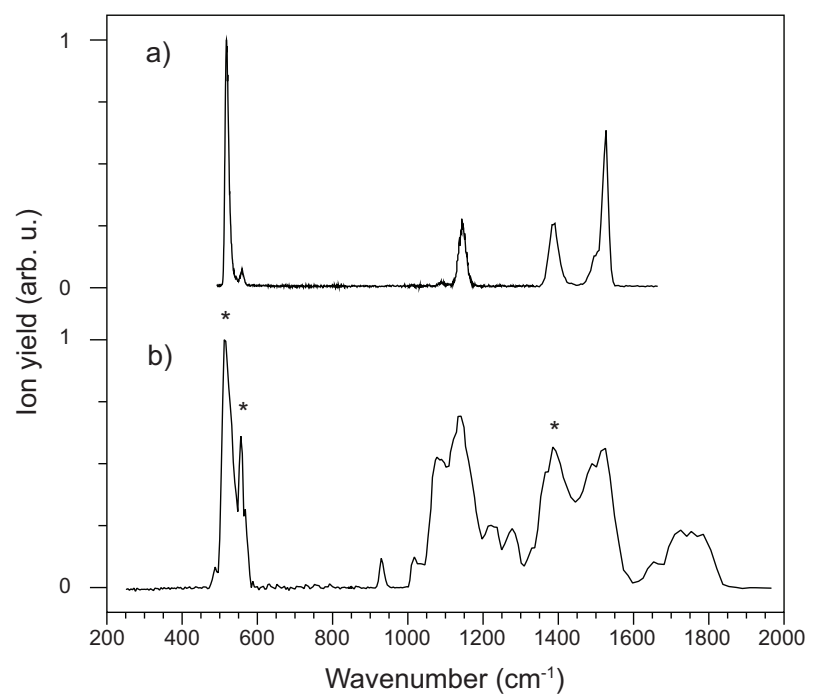

FIG. 2: IR spectra of gas-phase $\mathrm{C}_{60}$ molecules measured by recording the integrated ion distribution resulting from irradiation (a) with FELIX from Ref 9 and (b) FELICE. The asterisks indicate the fundamental bands that are studied in more detail in this paper. 


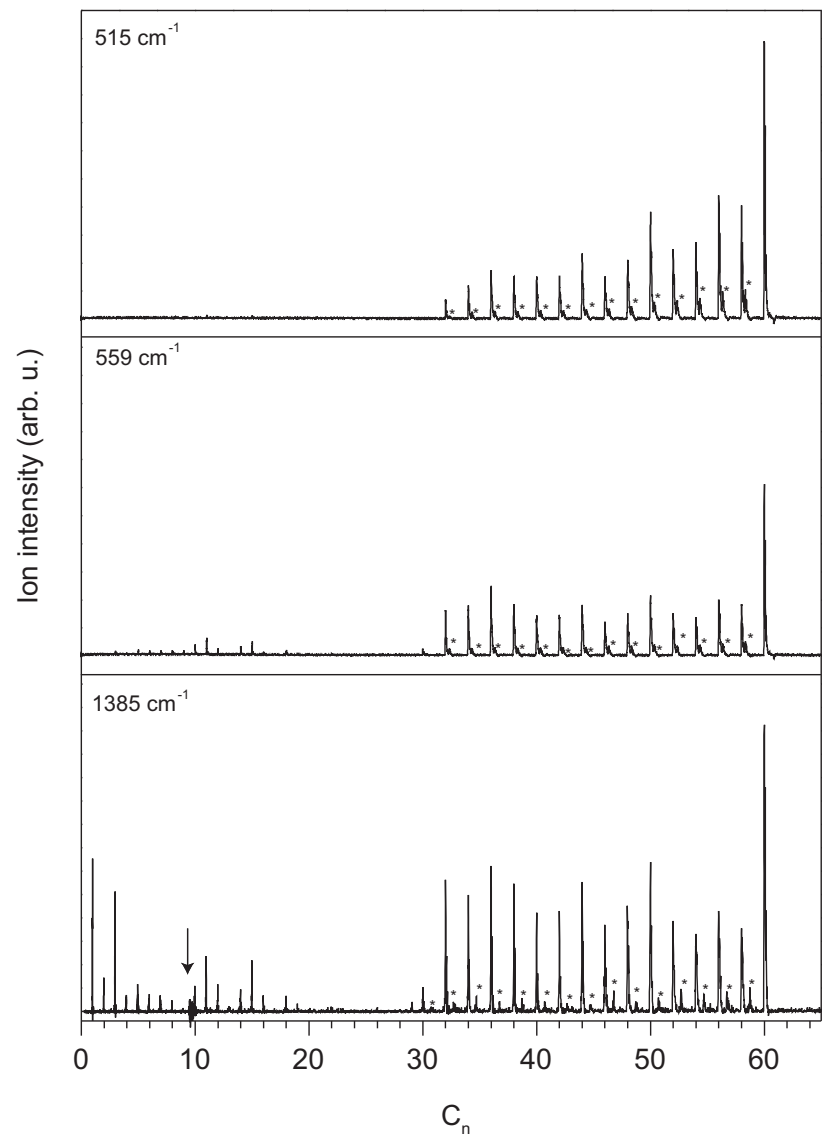

FIG. 3: Mass spectra of the ion distribution after interaction of neutral $\mathrm{C}_{60}$ molecules with one FELICE macropulse at 515, 559 and $1385 \mathrm{~cm}^{-1}$, respectively. Mass peaks indicated with an asterisk are caused by delayed fragmentation; an artifact due to pick-up of electrical noise is indicated with an arrow. 

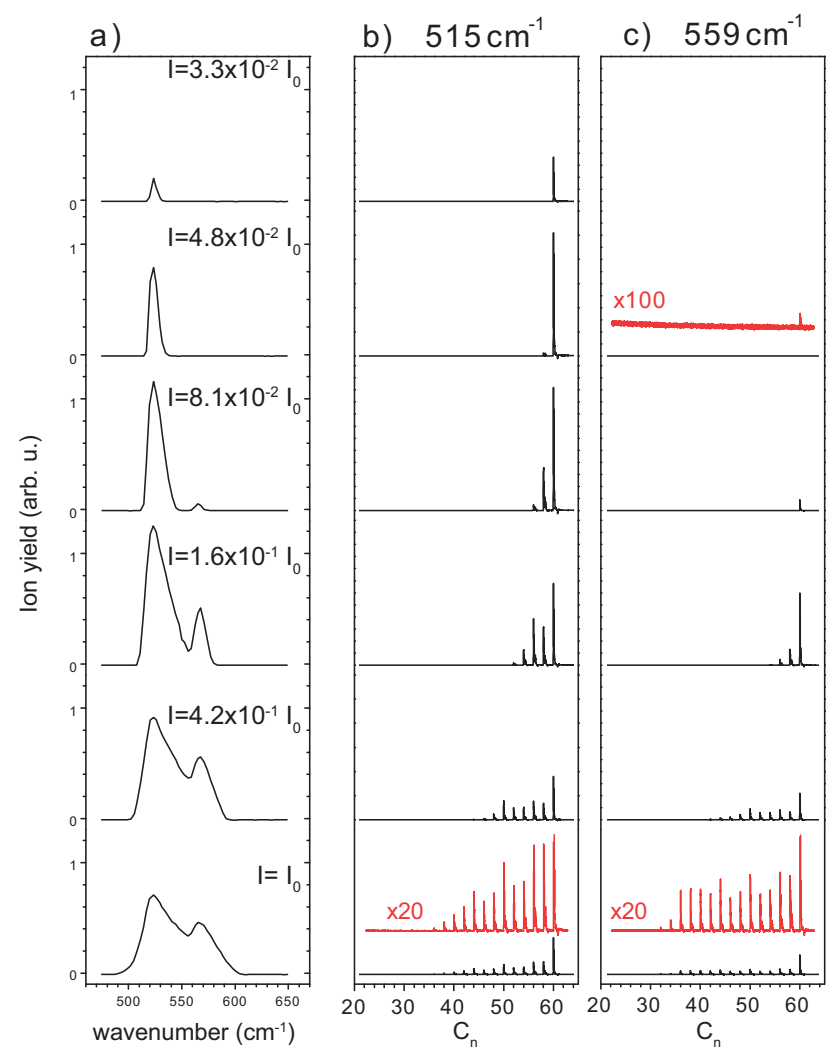

FIG. 4: Evolution of the IR action spectrum (column a) and the mass spectra for the resonances at $515 \mathrm{~cm}^{-1}$ (column b) and $559^{-1}$ (column c), respectively. The intensities are all on the same scale and not corrected for the interaction volume. 


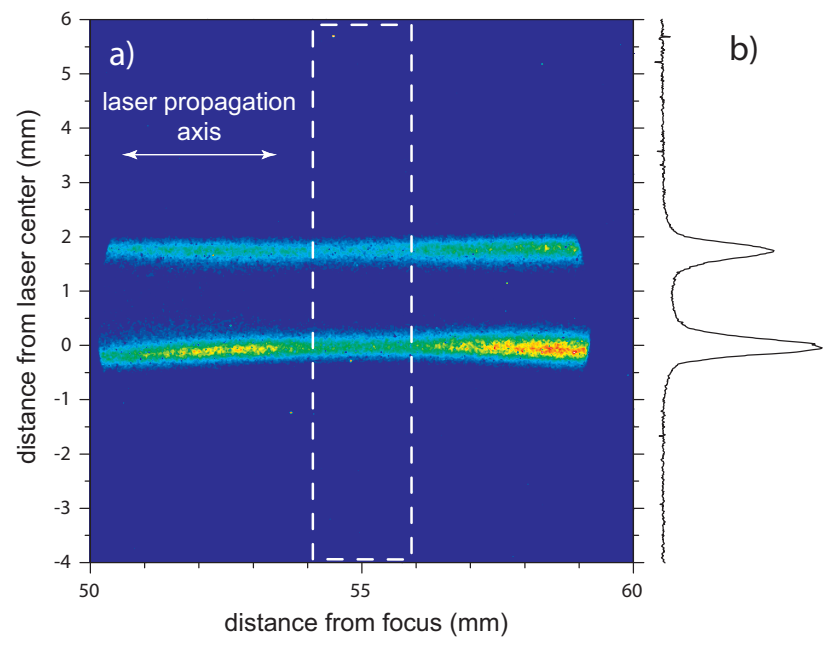

FIG. 5: Time-sliced spatial map image of the distribution of $\mathrm{C}_{60}^{+}$ions recorded at $528 \mathrm{~cm}^{-1}$. In panel (a) the raw recorded image is depicted while panel (b) shows the ion signal integrated along the laser propagation direction; the integration is performed over the center of the image as indicated by the square. 

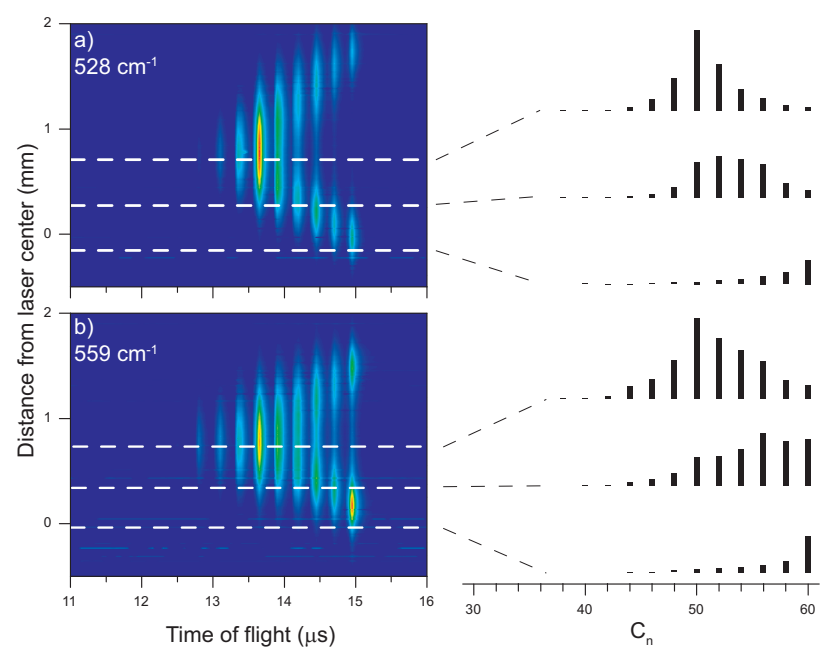

FIG. 6: Spatially resolved TOF mass spectra of ion distributions resulting from irradiation of neutral $\mathrm{C}_{60}$ molecules at $528 \mathrm{~cm}^{-1}$ (panel a) and $559 \mathrm{~cm}^{-1}$ (panel b), respectively, recorded at 55 $\mathrm{mm}$ from the focus of the laser beam. 


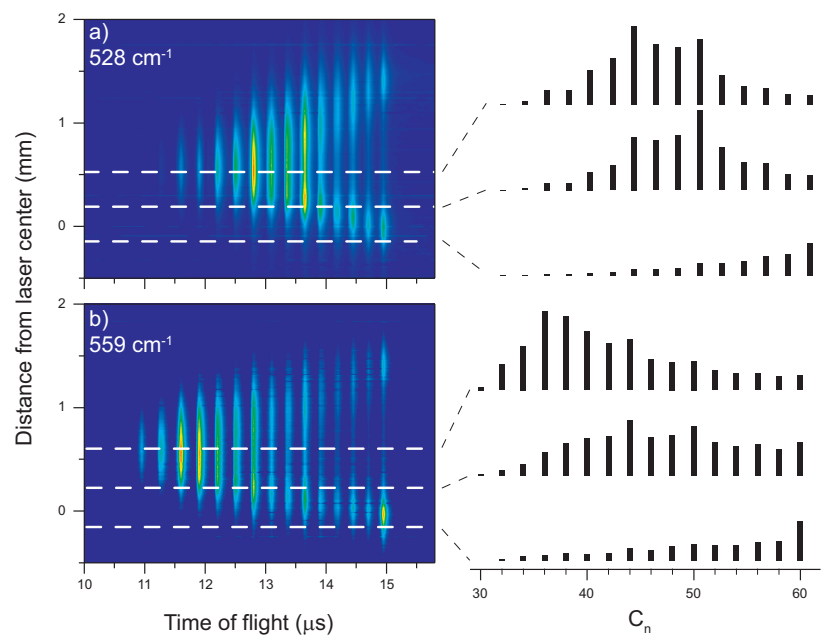

FIG. 7: Spatially resolved TOF mass spectra of ion distributions resulting from irradiation of neutral $\mathrm{C}_{60}$ molecules at $528 \mathrm{~cm}^{-1}$ (panel a) and $559 \mathrm{~cm}^{-1}$ (panel b), respectively, recorded at the focus of the laser beam. 


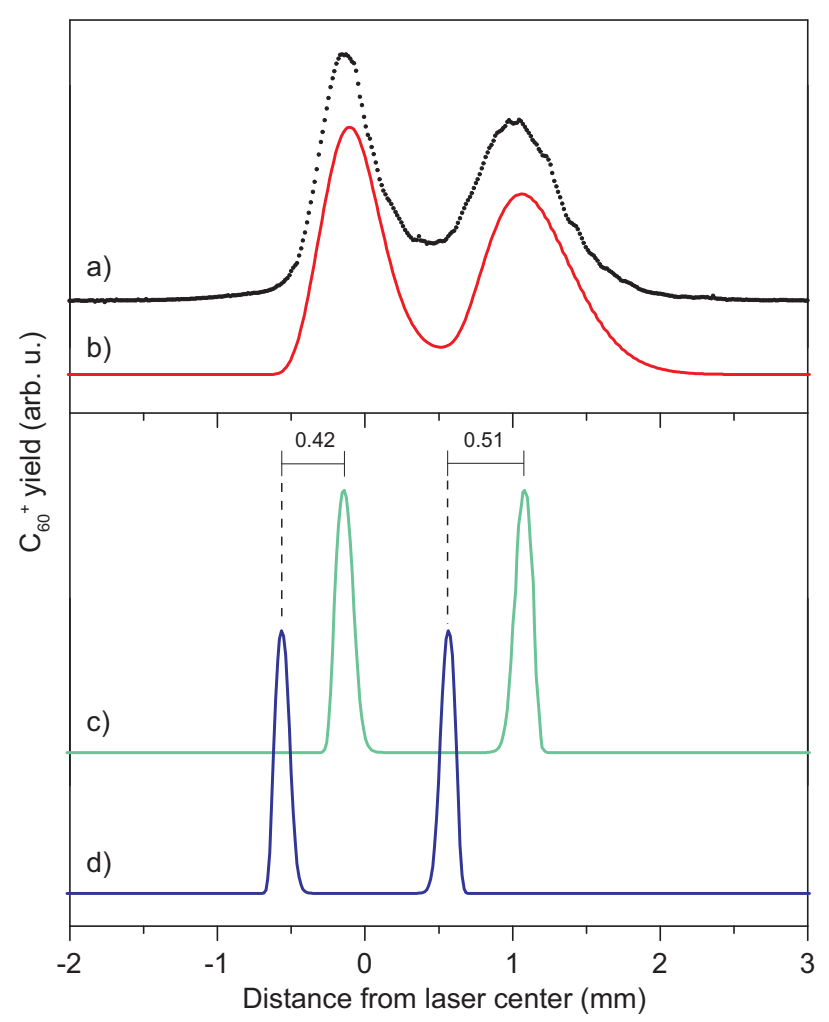

FIG. 8: (a) Observed spatial profile (integrated along the laser propagation axis) for ions resulting from IR-MP excitation of neutral $\mathrm{C}_{60}$ at $528 \mathrm{~cm}^{-1}$. Extraction is here $1 \mu \mathrm{s}$ after the end of the macropulse (b) Simulated profile assuming a Maxwellian velocity distribution. (c and d) Simulated profiles for molecules with a uniform velocity of 135 (c) and $0 \mathrm{~m} / \mathrm{s}$ (d), respectively. 


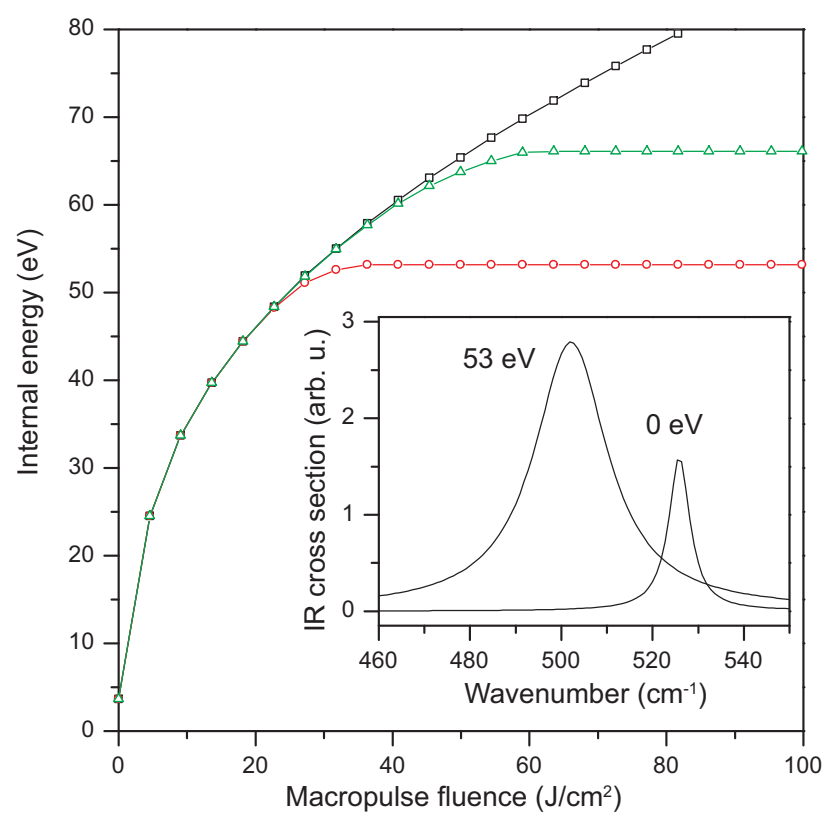

FIG. 9: Internal energy after IR-MP excitation of neutral $\mathrm{C}_{60}$ at $528 \mathrm{~cm}^{-1}$ excluding decay mechanisms (squares), including ionization only (triangles) and including ionization and fragmentation (circles). The inset shows the calculated spectral profile of the absorbing mode at an internal energy of 0 and $53 \mathrm{eV}$, respectively. 

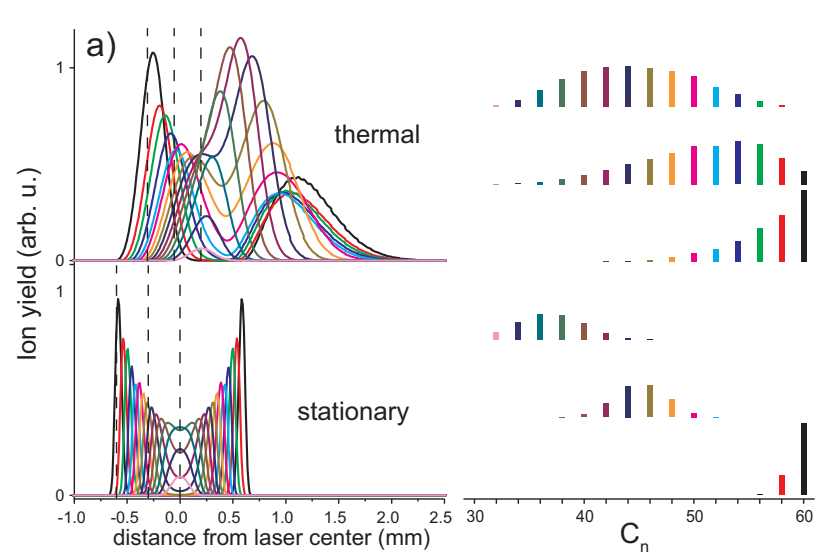

I II I. .
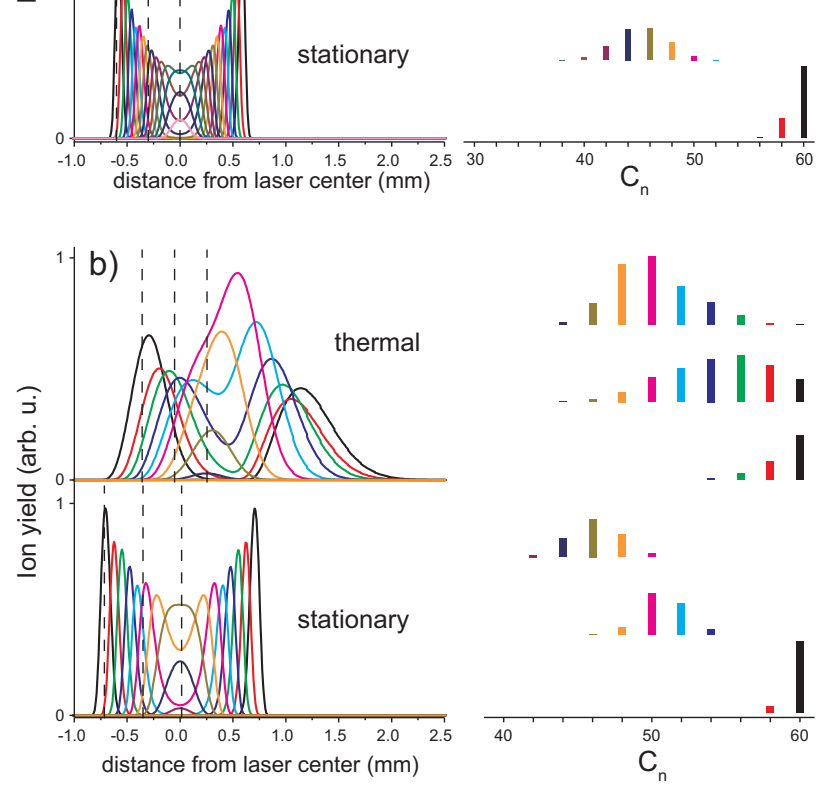

FIG. 10: Computed fragment distributions at the focus (panel a) and at $55 \mathrm{~mm}$ from the focus (panel b), both for the actual thermal velocity distribution and for zero velocity. The positions for which the mass distributions are shown on the right are indicated by dashed lines. 
Tables 
FELICE specifications relevant to these experiments

$\begin{array}{lc}\text { Wavenumber range } & 250-2000 \mathrm{~cm}^{-1} \\ \text { Energy per macropulse } & 0.5-5 \mathrm{~J} \\ \text { Macropulse length } & 4-5 \mu \mathrm{s} \\ \text { Macropulse repetition rate } & 5-10 \mathrm{~Hz} \\ \text { Energy per micropulse } & 0.1-1 \mathrm{~mJ} \\ \text { Micropulse repetition period } & 1 \mathrm{~ns} \\ \text { max. power density } & 3 \cdot 10^{13} / \lambda^{2} \mathrm{~W} / \mathrm{cm}^{2}(\lambda \text { in } \mu \mathrm{m}) \\ \text { Relative spectral bandwidth } & 0.4-2.0 \% \mathrm{FWHM} \\ \text { Rayleigh range } & 55 \mathrm{~mm}\end{array}$

TABLE I: Characteristics of the FELICE beam as used in the experiments described here. 